\title{
BARE CARBON/SILICATE THEORIES
}

\author{
JOHN S. MATHIS \\ Washburn Observatory, The University of Wisconsin-Madison \\ Madison, Wisconsin 59706, U. S. A.
}

\begin{abstract}
Several theories involving bare silicate grains, combined with carbon in either amorphous or graphitic form, are discussed and contrasted to the other grain theories outlined at this Symposium. The assumption of bare grains is justified, and power-law size distributions are strongly indicated from both theoretical and observational standpoints. Various successes and problems of these and other theories are discussed. All of the theories discussed in this Symposium can probably explain the observed extinction and polarization in general terms, but certain crucial observations, such as the wavelength behavior of the polarization and extinction in the wavelength range near the strong silicate absorption bands, will probably be able to discriminate among them.
\end{abstract}

\section{INTRODUCTION}

In this paper I will only consider dust grains in the diffuse interstellar medium or in the outer parts of dense clouds, rather than grains deep within dark clouds where there are icy mantles seen in absorption. Several theories of diffuse dust share common features: (a) They suggest that interstellar grains consist of mixtures of silicates, generally amorphous in nature, plus solid carbon in various forms (but they differ as to which form). None of the particles is assumed to have coatings or mantles, in contrast to the silicate core/organic-refractory-mantle theory (Greenberg and Hong, 1980; Greenberg, 1989). (b) They have particle size distributions which heavily favor small sizes, which is also a point of departure from Greenberg's theory. (c) They assume that the huge $2175 \AA$ feature (the "bump") is produced by small "graphite" particles. Here I use quotes around the "graphite" to mean merely a well-ordered form of carbon. True graphite is the limit of perfect order, but for the production of the bump a somewhat less ordered form would be sufficient. In this respect, these theories contrast with that of Duley and Williams (Williams, 1989).

\section{SEVERAL THEORIES OF BARE CARBON AND SILICATE GRAINS}

A theory using bare carbon and silicate grains (Mathis, Rumpl, and Nordsieck, 1977; hereafter MRN) was the first to produce a good quantitative fit to both the observed extinction law over the wavelength interval $0.12-1 \mu \mathrm{m}$. MRN showed that aurh a fit is nnesihle hv a varietv of erains of various compositions if each 
component has a power-law distribution of grain sizes, provided that graphite is present to produce the $2175 \AA$ bump. The number of particles per unit radius is assumed to be proportional to $a^{-3.5}$, where $a$ is the grain radius. The distribution is truncated at small sizes at about $0.01 \mu \mathrm{m}$ and at large sizes at about $0.25 \mu \mathrm{m}$. In subsequent papers (Mathis, 1979; Mathis and Wallenhorst, 1981; Mathis, 1986a), the MRN size distribution was assumed to include only graphite and silicates. In a very important paper, Draine and Lee (1984; hereafter DL) extended the wavelength range to which the MRN distribution was applied to explain by a factor of 1000 , to $1 \mathrm{~mm}$, and still found an adequate prediction of the observed extinction law. This extinction was in effect a prediction of the MRN mixture. This kind of fitting has been applied, to my knowledge, only to bare carbon/silicate theories.

Sellgren (1984) and Sellgren et al. (1985) discovered that diffuse dust in "reflection" nebulae produces the "Unidentified Infrared Bands" (UIRs). These bands are discussed very extensively in this conference from both observational and theoretical standpoints. Whatever their true origin, they are almost surely produced by very small particles (or fairly large molecules, by interstellar standards) which are radiating the energy acquired by absorption of a single ultraviolet, or possibly visible, photon. In order to account for the UIRs, Draine and Anderson (1985) extended the size distribution of DL down to molecular sizes. They found that they could account for the IRAS band emissivities if the exponent in the power-law distribution was steepened to 4 instead of 3.5. They calculated the band emissions by using bulk optical constants to connect the emissivity with the Planck function at a particular temperature. One can quarrel with their opacities for grains of molecular sizes, but if the band strengths for polycyclic aromatic hydrocarbons (PAHs) are used instead of the bulk optical constants, an excellent fit to the UIRs is obtained (Léger and Puget, 1984).

Dwek $(1986,1989)$ interpreted the IRAS emission from supernova remnants with a distribution of bare grains, the grains of which are heated by impacts with hot electrons. It is difficult to account for the emission and its spatial variation in any other way.

Through studies of the circumstellar dust surrounding carbon-rich stars (Greenstein, 1981; Hecht et al., 1984; Martin and Rogers, 1987), it became apparent that it is difficult to suppose that all solid carbon is formed as graphite, but rather that much of the carbon dust must be less well-ordered in form. Rowan-Robinson (1986) proposed a bare-grain theory which includes discrete sizes, both small (about 0.01 $\mu \mathrm{m}$ ) and large (about $0.1 \mu \mathrm{m}$ ) of amorphous silicates, small graphite grains (to provide the bump), and large amorphous carbon. Presumably, these discrete sizes represent a continuous distribution which is similar to a power-law (as can be seen by the relative masses contained in the various particles). The fit to the observed extinction law over the entire range of observed wavelengths is satisfactory.

There are problems with these bare carbon/silicate theories. They envisage large graphite grains, but I find it hard to believe that such highly-ordered and large pieces of graphite can be formed in the first place (Mathis, 1986b). They also suppose that grains stay either rather pure carbon or silicate. However, once grains are shattered, it is difficult to see how they could reform larger ones from smaller pieces and keep their compositions pure. It is somewhat difficult to believe on either observational or theoretical grounds that grains do not collide and coagulate while they are in dense regions within clouds. 


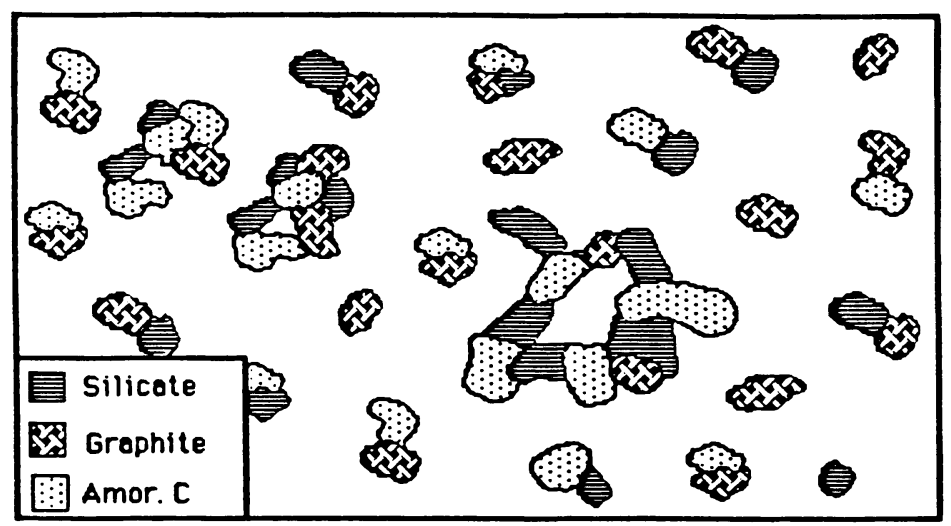

Fig. 1. A drawing showing proposed composite grains (Mathis and Whiffen, 1988) made of amorphous silicate, graphite, and amorphous carbon particles. Individual interstellar grains are assumed to consist of composite particles of all three constituents.

To avoid the difficulties with these bare carbon/silicate theories, I very recently proposed still another (Mathis and Whiffen, 1988). It consists of small particles of bare amorphous silicates, graphite, and amorphous carbon, all loosely packed together into composite grains with (perhaps) $80 \%$ of the volume of the grain consisting of vacuum. Figure 1 shows the assumed arrangement of small (about $50 \AA$ ) particles of amorphous silicate, amorphous carbon, and graphite within the composite grains. There is assumed to be a power-law size distribution for the composite grains themselves, with some free small particles of at least graphite (although small particles of either amorphous carbon or silicates cannot be excluded). Composite grains differ qualitatively from other bare carbon/silicate theories in that there is only kind of grain providing the extinction and polarization (except for the bump, arising from small graphite).

\subsection{WHY BARE GRAINS?}

Absorption bands of molecular ices of many common molecules are seen in the spectra of sources deep within cold molecular clouds, so everyone agrees that icy mantles form on the grains in such environments. As the Leiden group and others have shown (see e.g. Greenberg, 1986), these mantles should be heavily processed by UV radiation and/or high-energy cosmic ray particles. Subsequent warming of the mantles leaves a residue of "yellow stuff", stable at room temperatures, upon the silicate cores. The question relevant to dust in the diffuse interstellar medium (ISM) is: can "yellow stuff" survive the very rigorous processing which takes places behind interstellar shocks? The rate of survival of grains in the hostile postshock conditions is very sensitive to the binding energy of the materials (Draine, 1979; Draine and Salpeter, 1979). A material's being able to withstand heating to $300 \mathrm{~K}$ or even $500 \mathrm{~K}$ is certainly not enough to ensure that it will survive as well as the much more refractory solid carbon or silicates (which can be heated to well over 1000 $\mathrm{K})$. Current theories of grain destruction (McKee, 1989) suggest that it is not easy to understand how even carbon and silicate grains survive in the diffuse ISM (and 
it is known that in high-velocity clouds the silicates do not). It is much harder to imagine that "organic refractory" mantles can exist in such a hostile environment, unless they are processed to being highly carbonaceous themselves (see below).

Another reason, for having doubts that there are extensive organic refractory mantles on grains in the diffuse ISM, is that the mantles are not clearly seen locally. One signature of the organic refractory mantle is likely to be the aliphatic $C-H$ stretch at $3.4 \mu \mathrm{m}$ (not the $3.3 \mu \mathrm{m}$ aromatic $C-H$ which is seen in emission in the UIRs). This feature has been seen in the spectrum of IRS 7 near the galactic center (Butchart et al., 1985), where there is a weak ice feature at $3.1 \mu \mathrm{m}$ and about 30 magnitudes of visual extinction. However, in the nearby star VI Cyg No. 12, with $A(V)=10$ mag., there seems to be no detectable feature in the 3-3.4 $\mu \mathrm{m}$ region (Gillett et al., 1975). An absorption feature with the same ratio of optical depth to $A(V)$ as in IRS 7 should be readily visible (about a $10 \%$ absorption). It is hardly surprising that local dust seems not to have the same $3.4 \mu \mathrm{m}$ absorption strength per $A(V)$ as does the dust towards the galactic center, since the $O / H$ abundance ratio increases towards the center. Furthermore, there are probably dense clouds along the line of sight towards the center which promote the formation and retention of the organic residues. Even in the immediate vicinity of the VI Cyg No. 12, the dust has much greater than average spatial density, which should enhance the amount of any organic refractory mantles over the diffuse ISM. (Note: following the panel presentations, Dr. D. C. B. Whittet reported that he has recently reobserved VI Cyg 12. It does show a weak $3.4 \mu \mathrm{m}$ feature, with an optical depth per $A(V)$ of only $1 / 3$ of that in IRS 7 .)

There is a possibility that with very heavy processing, organic refractory mantles are indistinguishable from amorphous carbon. When subsequent grain-grain collisions shatter the core-mantle grains, there would be small particles of silicates and amorphous carbon which could coagulate into larger grains, exactly like bare carbon/silicates.

\subsection{WHY A POWER-LAW DISTRIBUTION?}

Another major point of departure of the core-mantle theory and the theories I am describing is the distribution of sizes. Figure 2 shows $n(a)$ for the MRN distribution and for core-mantle theory as given by Chlewicki and Greenberg (1988). The coremantle distribution is truncated at the lower end because of the assumed sizes of the silicate cores.

The normalization in the figure is that the integral over sizes of both distributions is unity. There are other versions of the core-mantle distributions which use larger particles in place of the one illustrated, but all have the quite flat shape as shown in Figure 2. The important feature shown in the figure is the very different relative numbers of small vs. larger particles in the two distributions. The area of the grains is determined by the small grains in the power-law distributions and by the larger ones in the core-mantle grains, while the mass of the grains is contained in the larger particles. It is the area which is of physical interest for several applications, such as: (a) the formation of molecular hydrogen, (b) the accretion of ices and formation of mantles, (c) the changes in size distribution, apparently mostly via coagulation of grains (see below), from the diffuse ISM as compared to the outer parts of molecular clouds, (d) the shattering of grains from grain-grain collisions, and (e) the heating of grains by impacts with energetic electrons in supernova remnants. 


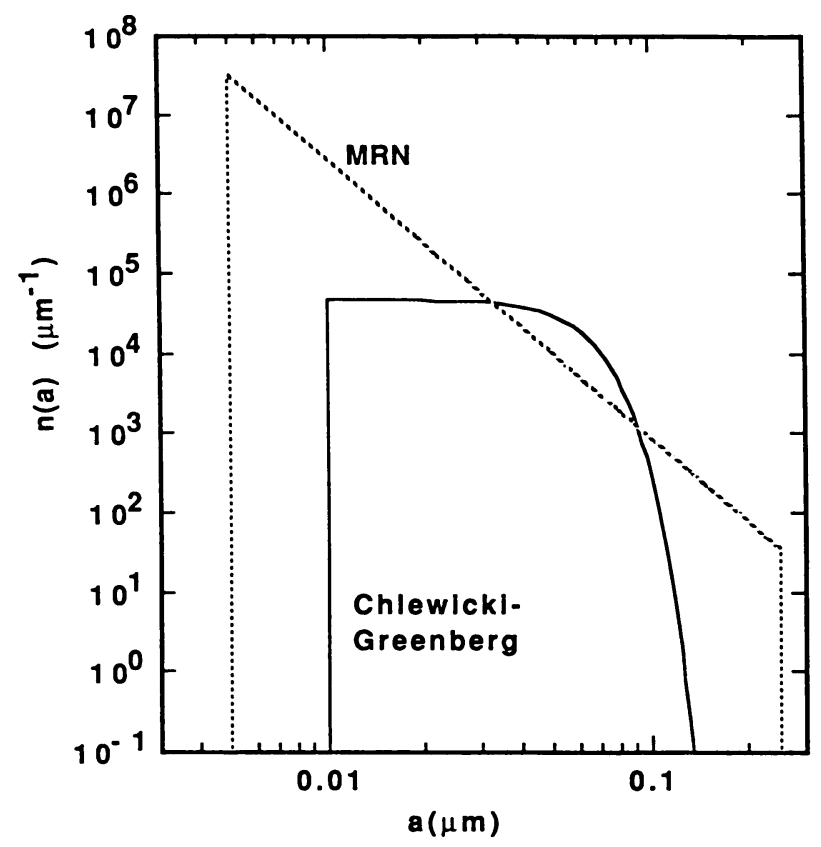

Fig. 2. The size distributions $n(a)$ for the MRN distribution and the core-mantle particles suggested by Chlewicki and Greenberg (1988), where $n(a) d a$ is the number of particles between radius $a$ and $a+d a$.

The core-mantle theory (Hong and Greenberg, 1980) does contain small particles (presumed to be silicates for the far-UV rise and small graphite for the bump) not shown in Figure 2 because their size distribution need not be specified as long as they are small in comparison with the wavelength of the bump. It is these small particles which determine the area of the grains for the core-mantle theory, rather than the core-mantle grains which have received almost all of the discussion from the Leiden group.

The case for the power-law distribution is very strong on both theoretical and observational grounds. Theoretically, it has been shown by Biermann and Harwit (1979) that the power-law is established by shattering of grains for the range of sizes in which there is a steady state; that is, the rate of producing a particular size from fragmenting larger particles is balanced by the rate of destruction of that size by shattering the grain into smaller particles. The largest and smallest sizes cannot be in such a steady state. The unphysical discontinuity assumed in the MRN distribution (Figure 2) reflects the breaking down of the power-law for the extreme sizes. Unfortunately, the area per mass of grains is largely determined by the distribution of the smallest particles, and is, therefore, somewhat uncertain. However, over most of the sizes one expects a power-law.

Observationally, power-law distributions are found in many diverse circumstances. Examples are the sizes of particles in the rings of Saturn (Cuzzi et al., 
1984), in the dust in the inner coma of Comet Halley as determined by Giotto's impact detectors (McDonnell et al., 1987), and in various kinds of terrestrial and lunar rocky and sandy debris (see e.g. Hartmann, 1969). The exponents of each of these power laws is near the range of MRN: exponents of $\mathbf{- 2 . 8}$ to -4.5 , while MRN suggested -3.5. These distributions are found whenever there is shattering and/or coagulation of grains through grain-grain collisions. Presumably, grains will undergo these processes within the dense regions of clouds in which they spend a substantial fraction of their time. It seems difficult to avoid steep power-law distributions for grains. Theories which do not favor them must justify their absence with some fairly convincing arguments.

\section{SUCCESSES AND PROBLEMS WITH BARE CARBON/SILICATE THEO- RIES}

\subsection{SUCCESSES}

I think that it is fair to say that all of the theories discussed in this conference (the core-mantle distribution of Greenberg, the Duley/Williams amorphous carbon/silicate, and the bare carbon/silicate theories) can probably be adjusted to fit the extinction data at a reasonable level of precision over most of the wavelengths available to observations. Since the alignment mechanism of interstellar grains is not well understood, the polarization data can probably be fitted fairly well too (see e. g. Hong and Greenberg, 1980; Mathis, 1986a; Williams, 1989) The basic reason for the agreement of the extinction is that the materials used in the theories, excepting the bump, are similar to amorphous carbon and silicates. However, there are certain key regions of the spectrum which should provide important tests for the correctness of the theories: for instance, the polarization in the spectral region around the silicate band $(8-11 \mu \mathrm{m})$ provides a powerful diagnostic as to the environment of the silicates in the ISM. The theories will also differ as regards the dielectric constants in the far-infrared (wavelengths $>20 \mu \mathrm{m}$ ).

Given the problems of deciding among theories on the basis of extinction and polarization predictions, we might decide on the basis of plausibility. I find the bare carbon/silicate theories satisfying for several reasons. (a) As mentioned above, the power-law seems to me to be hard to avoid for both theoretical and observational reasons. Also, there are questions regarding the importance of mantles on local dust. (b) Bare carbon/silicate theories explain in a rather natural and quantitative way the differences in extinction between the diffuse ISM and that found in the observable regions within dark clouds (Mathis and Wallenhorst, 1981; Mathis and Whiffen, 1988). (c) The bare carbon/silicate and core/mantle theories satisfy the cosmic abundance constraints by using graphite to provide the bump. The strength of the bump is discussed by Draine (1989). I feel that making the bump with elements less abundant than carbon requires an unreasonably high $f$-value for the transition and an abundance of the carrier which is unreasonably large in comparison with the abundance available. For example, the Duley/Williams theory uses $\mathrm{OH}^{-}$ions in connection with $S i$ atoms in silicates. (d) The sources of grains, primarily giant stars which are individually either carbon-rich or oxygenrich, are producing solid carbon and silicates, respectively. Small carbon grains can be processed into graphite by the repeated heating of the grain by single UV photons. Thus, the building blocks of the bare carbon/silicate theories can be made by plausible processes. 


\subsection{PROBLEMS}

There are certain difficulties common to all grain theories. A major source of uncertainty is the optical constants of the individual constituents. There is a continuum in the amount of graphitization which is possible in carbon, and also of the amount of diamond bonding relative to graphitic bonding. There are major uncertainties regarding the amount and nature of the hydrogenation of the amorphous carbon, and the amount of PAHs or other aromatic hydrocarbons present. Whatever is responsible for producing the UIRs must have strong UV absorption whose wavelength dependence is presently unknown. Because of these uncertainties, no one has yet included PAHs explicitly in a grain model, although they can be rather naturally incorporated into any of the theories under consideration at this conference by slightly broadening the definition of amorphous carbon or graphite.

The variation of optical properties with grain shape is another problem which has rather important implications for large-angle scattering by grains, but not so much for extinction. Fortunately, the approximation that grains behave very similarly to spheres of the same volume is quite good. For the theory of composite grains (Mathis and Whiffen, 1988), another difficulty enters: how to model a mixture of materials incorporated into the same grain. There are only approximate "mixing rules" available at present, but computationally cumbersome methods (the "Discrete Dipole Approximation") can circumvent this problem.

Another problem of all theories is the bump (Fitzpatrick and Massa, 1986, 1988; Cardelli and Savage, 1988; see also Draine, 1989). No present theory is able to explain all of its properties. Small graphite particles can account for its great strength, but not easily for the small, but real, variations in central wavelength. Its width, which varies by almost a factor of two, is not correlated to other properties of the extinction or environment except that it is broader in regions of large mean gas density. Small graphite alone will not account for these observations. Perhaps there are either impurities (Hecht, 1987), surface coatings, or shape changes which slightly shift the optical properties from one line of sight to another. These loose statements do not qualify as a "theory" of the bump. I am concerned that graphitic carbon is very rare in meteorites (Nuth, 1985).

The composite grain theory has its share of problems: Are grains strong enough to spin enough to align themselves? Are there enough grain-grain collisions which result in coagulation into larger composite grains? What is the proper fraction of vacuum to assume for each grain size? (Here I am thinking of the possibility of fractal grains, which would have a size-dependent fluffy structure.)

There is another problem, concerned with the variation of $A(V) / N(H)$ with grain size. The grains size can be characterized by $R_{V}=A(V) / E(B-V)$. It is possible to estimate the neutral hydrogen column density along various lines of sight from the wings of Lyman-alpha as determined by the International Ultraviolet Explorer satellite (Shull and van Steenberg, 1985), and of molecular hydrogen from the Copernicus satellite (Savage et al., 1977). There are additional measurements of $\rho$ Oph (de Boer et al., 1986). The mean value of $A(V) / N(H)$ for the diffuse ISM (where $\left.R_{V}=3.1\right)$ is $5.3 \times 10^{-22} \mathrm{mag}-\mathrm{cm}^{2}-(H \text { atom) })^{-1}$ (Bohlin, Savage, and Drake, 1978).

The observations of $A(V) / N(H)$ for individual stars show at least two stars of large $R_{V}$ ( $\rho$ Oph and $\nu$ Ori) which definitely seem to have smaller than average 
values. The uncertainties in the other measurements are too large to provide believable general trends, but a viable grain theory should at least be able to explain the observations for the well determined line of sight to $\rho$ Oph. The composite grain theory predicts an increase of $A(V) / N(H)$ with $R_{V}$ by a factor of 1.2 to $\rho$ Oph. Any theory (the core-mantle, for instance) which predicts that the increase of $R_{V}$ in dense clouds is caused by accretion of additional mantles would predict a larger increase of $A(V) / N(H)$ than the composite grains, and would be even more discrepant with the observations.

Another test was suggested (Martin, 1989; also Hildebrand during the discussion following the presentation): the polarization of $\mathrm{BN}$ in the $9.7 \mu \mathrm{m}$ region places stringent limits (Lee and Draine, 1985) on the shapes and very local environment of the silicates (i.e., mantles or neighboring particles). Hildebrand claimed that mantles are apparently excluded by his calculations (another severe difficulty for the core-mantle theory). Composite grains also have the silicates in close proximity to conductors (i.e., graphite). Unfortunately, there are problems in adding the dielectric constants of diverse materials, so no unambiguous test is presently possible. Since the composite grains are $80 \%$ vacuum, it is possible that they can pass this test, but it is surely possible that they cannot. The Duley-Williams theory also has carbon in close proximity to the silicates, so perhaps the only survivors of this test will be those which separate carbon and silicates into different grains (DL, Dwek, Rowan-Robinson, etc.).

Theories are pretty slippery. They have a way of accommodating to new observations which at first sight appear to be fatal. Another common approach is hoping that the observations themselves will disappear, as they sometimes do. I hope that some of the present tests will be able to narrow the field of acceptable grain theories in the near future.

ACKNOWLEDGEMENTS. This research has partly been supported by NASA through its contract 957996, and by NSF by its grant AST-8415142. I have benefitted with conversations with many colleagues, some of whom did not share my views.

\section{REFERENCES}

Biermann, P., and Harwit, M. 1979, Ap. J., 241, L33.

Butchart, I., McFadzean, A. D., Whittet, D. C. B., Geballe, T. R., and Greenberg, J. M. 1985, Astr. Ap., 154, L5.

Cardelli, J. A., and Savage, B. D. 1988, Ap. J., 825, 864.

Chlewicki, G., and Greenberg, J. M. 1988, Ap. J., in press.

Cussi, J. N., Lissauer, J. J., Esposito, L. W., Holberg, J. B., Marouf, E. A., Tyler, G. L., and Boischot, A. 1984, in Planetary Rings, eds. R. G. Greenberg and A. Brahic, (Tucson: U of AZ Pr.), p. 73.

de Boer, K. S., Lenhart, H., van der Hucht, K. A., Kamperman, T. M., Kondo, Y., and Bruhweiler, F. C. 1986, Astr. Ap., 157, 119.

Draine, B. T. 1979, Ap. J., 231, 438.

1989 , in IAU Symposium 195, Interstellar Dust, eds. L. J. Allamandola and A. G. G. M. Tielens, (Dordrecht: Kluwer), p. 313.

Draine, B. T., and Anderson, N. 1985, Ap. J., $292,494$.

Draine, B. T., and Lee, H. M. 1984, Ap. J., 285, 89. 
Draine, B. T., and Salpeter, E. E. 1979, Ap. J., $231,77$.

Duley, W. W., and Williams, D. A. 1988, M. N. R. A. S., $231,969$.

Dwek, E. 1989 in IAU Symposium 195, Interstellar Dust, eds. L. J. Allamandola and A. G. G. M. Tielens, (Dordrecht: Kluwer), p. 479. . 1986, A p. J., 802, 363.

Fitzpatrick, E. L., and Massa, D. 1986, Ap. J., 307, 286.

. 1988, Ap. J., 328, 734 .

Gillett, F. C., Jones, T. W., Merrill, K. M., and Stein, W. A. 1975, Astr. Ap., 45, 77.

Greenberg, J. M. 1986, in Light on Dark Matter, ed. F. P. Israel, (Dordrecht: Reidel), p. 177.

- 1989, in IAU Symposium 195, Interstellar Dust, eds. L. J. Allamandola and A. G. G. M. Tielens, (Dordrecht: Kluwer), p. 345.

Greenstein, J. L. 1981, Ap. J., 245, 124.

Hartmann, W. K. 1969, Icarus, 10, 201.

Hecht, J. H. 1987, Ap. J., \$14, 429.

Hecht, J. H., Holm, A. V., Donn, B., and Wu, C. -C. 1984, Ap. J., 280, 228.

Hong, S. S., and Greenberg, J. M. 1980, Astr. Ap., 88, 194.

Lee, H. M., and Draine, B. T. 1985, Ap. J., 290, 85.

Léger, A., and Puget, J. L. 1984, Astr. Ap., 137, L5.

Martin, P. G. 1989, in IAU Symposium 195, Interstellar Dust, eds. L. J. Allamandola and A. G. G. M. Tielens, (Dordrecht: Kluwer), p. 375.

Mathis, J. S. $1986 a, A p$. J., 308, 281.

1986 , in Light on Dark Matter, ed. F. P. Israel, (Dordrecht: Reidel), p. 171.

Mathis, J. S., and Wallenhorst, S. G. 1981, Ap. J., 244, 483.

Mathis, J. S., and Whiffen, G. 1988, Ap. J., submitted.

Mathis, J. S., Rumpl. W., and Nordsieck, K. H. 1977, Ap. J., 217, 425 (MRN).

McDonnell, J. A. M., et al. 1987, Astr. Ap., 187, 719 .

McKee, C. F. 1989, in IAU Symposium 195, Interstellar Dust, eds. L. J. Allamandola and A. G. G. M. Tielens, (Dordrecht: Kluwer), p. 431.

Nuth, J. A. 1985, Nature, 818, 166.

Rowan-Robinson, M. 1986, M. N. R. A. S., 219, 737.

Sellgren, K. 1984, Ap. J., 277, 623.

Sellgren, K., Allamandola, L. J., Bregman, J. D., Werner, M. W., and Wooden, D. H. 1985, Ap. J., $299,416$.

Shull, J. M., and van Steenberg, M. E. 1985, Ap. J., 294, 599.

Williams, D. A. 1989 in IAU Symposium 195, Interstellar Dust, eds. L. J. Allamandola and A. G. G. M. Tielens, (Dordrecht: Kluwer), p. 367. 